\title{
AUTOMORPHISMS OF CUBIC SURFACES WITHOUT POINTS
}

\author{
CONSTANTIN SHRAMOV
}

\begin{abstract}
We classify finite groups acting by birational transformations of a nontrivial Severi-Brauer surface over a field of characteristc zero that are not conjugate to subgroups of the automorphism group. Also, we show that the automorphism group of a smooth cubic surface over a field $\mathbb{K}$ of characteristic zero that has no $\mathbb{K}$-points is abelian, and find a sharp bound for the Jordan constants of birational automorphism groups of such cubic surfaces.
\end{abstract}

\section{INTRODUCTION}

Given a variety $X$, it is natural to try to describe finite subgroups of its birational automorphism group $\operatorname{Bir}(X)$ in terms of the birational models of $X$ on which these finite subgroups are regularized. In dimension 2, this is sometimes possible due to the well developed Minimal Model Program. In the case of the group of birational automorphisms of the projective plane over an algebraically closed field of characteristic zero, this was done in the work of I. Dolgachev and V. Iskovskikh [DI09]. Over algebraically non-closed fields, some partial results are also known for non-trivial Severi-Brauer surfaces, i.e. del Pezzo surfaces of degree 9 not isomorphic to $\mathbb{P}^{2}$ over the base field. Note that the latter surfaces are exactly the del Pezzo surfaces of degree 9 without points over the base field, see e.g. [Ko16, Theorem 53(2)].

Recall that a birational map $Y \rightarrow X$ defines an embedding of a group $\operatorname{Aut}(Y)$ into $\operatorname{Bir}(X)$. We will denote by $\operatorname{rk} \operatorname{Pic}(X)^{\Gamma}$ the invariant part of the Picard group $\operatorname{Pic}(X)$ with respect to the action of a group $\Gamma$, and by $\boldsymbol{\mu}_{n}$ the cyclic group of order $n$. The following partial description of finite groups of birational automorphisms of non-trivial Severi-Brauer surfaces is known.

Proposition 1.1 ([Sh20a, Proposition 3.7], [Sh20a, Corollary 4.5]). Let $S$ be a nontrivial Severi-Brauer surface over a field $\mathbb{K}$ of characteristic zero, and let $G \subset \operatorname{Bir}(S)$ be a finite subgroup. Then $G$ is conjugate either to a subgroup of $\operatorname{Aut}(S)$, or to a subgroup of $\operatorname{Aut}\left(S^{\prime}\right)$, where $S^{\prime}$ is a smooth cubic surface over $\mathbb{K}$ birational to $S$ such that $\mathrm{rk} \operatorname{Pic}\left(S^{\prime}\right)=3$ and $\operatorname{rk} \operatorname{Pic}\left(S^{\prime}\right)^{G}=1$. In the latter case $G$ is isomorphic to a subgroup of $\boldsymbol{\mu}_{3}^{3}$.

However, it was not known whether the second of the two cases listed in Proposition 1.1 can indeed occur. The first goal of this paper is to construct such examples and thus complete the classification of finite subgroups of birational automorphism groups of non-trivial Severi-Brauer surfaces that are not conjugate to subgroups of automorphism groups.

Theorem 1.2. Let $G$ be one of the groups $\boldsymbol{\mu}_{3}, \boldsymbol{\mu}_{3}^{2}$, and $\boldsymbol{\mu}_{3}^{3}$. Then there exists a field $\mathbb{K}$ of characteristic zero and a non-trivial Severi-Brauer surface $S$ over $\mathbb{K}$ such that $\operatorname{Bir}(S)$ contains a subgroup isomorphic to $G$ and not conjugate to a subgroup of $\operatorname{Aut}(S)$.

This work is supported by the Russian Science Foundation under grant No. 19-11-00237. 
Proposition 1.1 was used in Sh20a to study finite subgroups of birational automorphism groups of non-trivial Severi-Brauer surfaces.

Theorem 1.3 ([Sh20a, Theorem 1.2(ii)], [Sh20b]). Let $\mathbb{K}$ be a field of characteristic zero, and let $S$ be a non-trivial Severi-Brauer surface over $\mathbb{K}$. Let $G$ be a finite subgroup of $\operatorname{Bir}(S)$. Then $G$ is either abelian, or contains a normal abelian subgroup of index 3 . Furthermore, there exists a field $\mathbb{K}_{0}$ of characteristic zero, a non-trivial Severi-Brauer surface $S_{0}$ over $\mathbb{K}_{0}$, and a finite subgroup $G_{0} \subset \operatorname{Aut}\left(S_{0}\right)$ such that the minimal index of a normal abelian subgroup in $G_{0}$ equals 3 .

Using the notion of Jordan constant (see [Po14, Definition 1]), one can reformulate Theorem 1.3 by saying that the Jordan constant of the birational automorphism group of a non-trivial Severi-Brauer surface over a field of characteristic zero does not exceed 3, and this bound is attained over suitable fields. We refer the reader to [Ya17, Theorem 1.9] for an analog of this result for the group of birational automorphisms of the projective plane.

As a by-product of the constructions used in this paper, we prove an analog of Theorem 1.3 for automorphism groups of cubic surfaces without points over the base field (including those that are not birational to any Severi-Brauer surface).

Theorem 1.4. Let $\mathbb{K}$ be a field of characteristic zero, and let $S$ be a smooth cubic surface over $\mathbb{K}$. Suppose that $S$ has no $\mathbb{K}$-points. The following assertions hold.

(i) The group $\operatorname{Aut}(S)$ is abelian.

(ii) Every finite subgroup of $\operatorname{Bir}(S)$ has a normal abelian subgroup of index at most 3 .

(iii) There exists a field $\mathbb{K}_{0}$ of characteristic zero, a smooth cubic surface $S_{0}$ over $\mathbb{K}_{0}$ with $S_{0}\left(\mathbb{K}_{0}\right)=\varnothing$, and a finite subgroup $G_{0} \subset \operatorname{Bir}\left(S_{0}\right)$ such that the minimal index of a normal abelian subgroup in $G_{0}$ equals 3 .

In other words, if $S$ is a smooth cubic surface over a field $\mathbb{K}$ of characterisitic zero with $S(\mathbb{K})=\varnothing$, then the Jordan constant the group $\operatorname{Bir}(S)$ equals 3 , and this bound is attained for certain cubic surfaces over suitable fields.

In course of the proof of our main results, we obtain a classification of birational models of smooth cubic surfaces without points and del Pezzo surfaces of degree 6 without points of degree 1 and 2 that we find interesting on its own.

Proposition 1.5. Let $\mathbb{K}$ be a field of characteristic zero, and let $S$ be a surface over $\mathbb{K}$ of one of the following three types:

- a smooth cubic surface with no $\mathbb{K}$-points;

- a del Pezzo surface of degree 6 with no points of degree 1 and 2 over $\mathbb{K}$;

- a non-trivial Severi-Brauer surface.

Suppose that $S^{\prime}$ is a surface over $\mathbb{K}$ birational to $S$, such that $S^{\prime}$ is either a del Pezzo surface or a conic bundle. Then $S^{\prime}$ is also a surface of one of the above three types. In particular, $S^{\prime}$ is not a conic bundle.

The plan of the paper is as follows. In Section 2 we prove some auxiliary results concerning birational geometry of cubic surfaces. In Section 3 we provide a construction that proves Theorem 1.2. In Section 4 we discuss automorphisms of plane cubic curves. In Section 5 we prove the first assertion of Theorem [1.4. In Section 6] we make some additional observations on del Pezzo surfaces of degree 6. In Section 7 we complete the proof of Theorem 1.4. 
Notation and conventions. We denote by $\mathfrak{S}_{n}$ the symmetric group on $n$ letters.

Given a field $\mathbb{K}$, we denote by $\overline{\mathbb{K}}$ its algebraic closure. If $X$ is a variety defined over $\mathbb{K}$ and $\mathbb{L}$ is an extension of $\mathbb{K}$, we denote by $X_{\mathbb{L}}$ the extension of scalars of $X$ to $\mathbb{L}$. By a point of degree $r$ on a variety defined over some field $\mathbb{K}$ we mean a closed point whose residue field is an extension of $\mathbb{K}$ of degree $r$; a $\mathbb{K}$-point is a point of degree 1 . The set of all $\mathbb{K}$-points of $X$ is denoted by $X(\mathbb{K})$.

A del Pezzo surface is a smooth projective surface with an ample anticanonical class. For a del Pezzo surface $S$, by its degree we mean its (anti)canonical degree $K_{S}^{2}$. Del Pezzo surfaces of degree 3 are exactly smooth cubic surfaces.

Acknowledgements. I am grateful to A. Trepalin and V. Vologodsky for useful discussions, and to J.-L. Colliot-Thélène for interesting references.

\section{Birational Models of CUBiC SURFACES}

The following assertion is known as the theorem of Lang and Nishimura.

Theorem 2.1 (see e.g. [VA13, Lemma 1.1]). Let $X$ and $Y$ be smooth projective varieties over an arbitrary field $\mathbb{K}$. Suppose that $X$ is birational to $Y$. Then $X$ has a $\mathbb{K}$-point of degree at most $d$ if and only if $Y$ has a $\mathbb{K}$-point of degree at most $d$.

It appears that a cubic surface without $\mathbb{K}$-points cannot be birationally transformed to del Pezzo surfaces of degrees 1, 2, 4, 5, 7, and 8 .

Lemma 2.2. Let $S$ be a smooth cubic surface over a field $\mathbb{K}$ of characteristic zero with $S(\mathbb{K})=\varnothing$. Then $S$ has no points of degree 2 over $\mathbb{K}$.

Proof. Suppose that $S$ contains a point $P$ of degree 2 over $\mathbb{K}$. Let $L$ be the line in $\mathbb{P}^{3}$ passing through $P$; then $L$ is defined over $\mathbb{K}$ as well. Note that $L$ always has a $\mathbb{K}$-point. If $L$ is contained in $S$, then $S$ has a $\mathbb{K}$-point. If $L$ is not contained in $S$, then the third intersection point of $L_{\overline{\mathbb{K}}}$ with $S_{\overline{\mathbb{K}}}$ is $\operatorname{Gal}(\overline{\mathbb{K}} / \mathbb{K})$-invariant and thus is defined over $\mathbb{K}$.

Corollary 2.3. Let $S$ be a smooth cubic surface over a field $\mathbb{K}$ of characteristic zero with $S(\mathbb{K})=\varnothing$. Then $S$ is not birational to del Pezzo surfaces of degree 1 and 2 .

Proof. A del Pezzo surface of degree $d$ over $\mathbb{K}$ always has a $\mathbb{K}$-point of degree at most $d$. Thus the assertion follows from Theorem 2.1 and Lemma 2.2.

Lemma 2.4. Let $S$ be a smooth cubic surface over a field $\mathbb{K}$ of characteristic zero with $S(\mathbb{K})=\varnothing$. Then $S$ is not birational to del Pezzo surfaces of degree 4 .

Proof. Suppose that $S$ is birational to a del Pezzo surface $S^{\prime}$ of degree 4 . Then $S^{\prime}$ has no $\mathbb{K}$-points by Theorem 2.1. However, $S$ has a point of degree 3 over $\mathbb{K}$, and thus Theorem 2.1 implies that $S^{\prime}$ also has a point $P$ of degree 3 . In this case it is known that $S^{\prime}$ has a $\mathbb{K}$-point, see [Co77] (alternatively, this can be deduced from a more general theorem due to Amer, Brumer, and Leep, see [EKM08, Theorem 17.14]). In spite of this, we prefer to give an elementary proof.

Let $P_{1}, P_{2}$, and $P_{3}$ be the three points of $P_{\overline{\mathbb{K}}}$. Consider the anticanonical embedding $S^{\prime} \hookrightarrow \mathbb{P}^{4}$. Suppose that the points $P_{1}, P_{2}$, and $P_{3}$ are collinear, and consider the line $L$ in $\mathbb{P}_{\mathbb{K}}^{4}$ passing through them. Then $L$ is $\mathrm{Gal}(\overline{\mathbb{K}} / \mathbb{K})$-invariant and thus defined over $\mathbb{K}$. Since it has at least three common points with the surface $S^{\prime}$, and $S^{\prime}$ can be represented as an intersection of two quadrics in $\mathbb{P}^{4}$, we conclude that $L$ is contained in $S^{\prime}$. Since $L$ has a $\mathbb{K}$-point, we see that $S^{\prime}$ has a $\mathbb{K}$-point as well, which gives a contradiction. 
Therefore, the points $P_{1}, P_{2}$, and $P_{3}$ are not collinear. Let $\Pi$ be the plane in $\mathbb{P}_{\mathbb{K}}^{4}$ passing through these three points. Then $\Pi$ is $\operatorname{Gal}(\overline{\mathbb{K}} / \mathbb{K})$-invariant and thus defined over $\mathbb{K}$. If the intersection $S_{\overline{\mathbb{K}}}^{\prime} \cap \Pi$ is zero-dimensional, it is easy to see that it consists of $P_{1}, P_{2}, P_{3}$, and one more point $P_{4}$. The point $P_{4}$ is $\operatorname{Gal}(\overline{\mathbb{K}} / \mathbb{K})$-invariant and thus defined over $\mathbb{K}$. If $C=S_{\mathbb{K}}^{\prime} \cap \Pi$ is one-dimensional, then the union of its one-dimensional irreducible components is either a line $L$, or a pair of coplanar lines $L_{1} \cup L_{2}$, or an irreducible conic. In the first case, the line $L$ is defined over $\mathbb{K}$, and thus contains a $\mathbb{K}$-point. In the second case, the point $L_{1} \cap L_{2}$ is defined over $\mathbb{K}$. In the third case, the conic $C$ is defined over $\mathbb{K}$ and contains the point $P$ of degree 3 over $\mathbb{K}$, which implies that it also contains a $\mathbb{K}$-point. In every possible case, we see that $S^{\prime}$ has a $\mathbb{K}$-point, which gives a contradiction.

Lemma 2.5. Let $S$ be a smooth cubic surface over a field $\mathbb{K}$ of characteristic zero with $S(\mathbb{K})=\varnothing$. Then $S$ is not birational to del Pezzo surfaces of degree 5 and 7 .

Proof. A del Pezzo surface of degree 5 or 7 over $\mathbb{K}$ always has a $\mathbb{K}$-point, see for instance [VA13, Theorem 2.5]. Thus the assertion follows from Theorem 2.1.

Lemma 2.6. Let $S$ be a smooth cubic surface over a field $\mathbb{K}$ of characteristic zero with $S(\mathbb{K})=\varnothing$. Then $S$ is not birational to a del Pezzo surface of degree 8 .

Proof. Suppose that $S$ is birational to a del Pezzo surface $S^{\prime}$ of degree 8. Then $S^{\prime}$ has no points of degree 1 and 2 over $\mathbb{K}$ by Theorem 2.1 and Lemma 2.2. If $S_{\overline{\mathbb{K}}}^{\prime}$ is not isomorphic to $\mathbb{P}^{1} \times \mathbb{P}^{1}$, then $S$ contains a $(-1)$-curve defined over $\mathbb{K}$; this implies that $S^{\prime}$ has a $\mathbb{K}$-point, which gives a contradiction. Therefore, we see that $S_{\overline{\mathbb{K}}}^{\prime} \cong \mathbb{P}^{1} \times \mathbb{P}^{1}$. Hence the surface $S^{\prime}$ is isomorphic either to a product $C_{1} \times C_{2}$, where $C_{1}$ and $C_{2}$ are conics over $\mathbb{K}$, or to the Weil restriction of scalars $R_{\mathbb{L} / \mathbb{K}} Q$ of a conic $Q$ defined over some quadratic extension $\mathbb{L}$ of $\mathbb{K}$, see e.g. [ShV18, Lemma 7.3(i)].

Note that $S$ always has a point of degree 3 over $\mathbb{K}$. By Theorem 2.1 , this implies that $S^{\prime}$ has a point of degree 1 or 3 over $\mathbb{K}$. Since $S^{\prime}$ has no $\mathbb{K}$-points, we conclude that it has a $\mathbb{K}$-point of degree 3 . If $S^{\prime} \cong C_{1} \times C_{2}$, then each of the conics $C_{i}$ contains a point of odd degree over $\mathbb{K}$, and hence $C_{1} \cong C_{2} \cong \mathbb{P}^{1}$. This means that $S^{\prime}$ has a $\mathbb{K}$-point, which gives a contradiction. If $S^{\prime} \cong R_{\mathbb{L} / \mathbb{K}} Q$, then the conic $Q$ contains a point of degree 3 over $\mathbb{L}$, and hence is isomorphic to $\mathbb{P}^{1}$. Thus $Q$ has an $\mathbb{L}$-point, and so $S^{\prime}$ has a $\mathbb{K}$-point, which again gives a contradiction.

Remark 2.7. If $S$ is a smooth cubic surface over a field $\mathbb{K}$ of characteristic zero with $S(\mathbb{K})=\varnothing$, then $S$ is not birational to any surface with a point of degree at most 2 over $\mathbb{K}$ by Lemma 2.2 and Theorem 2.1. Therefore, in some cases the assertion of Lemma 2.6 can be deduced from the existence of such a point on the del Pezzo surface $S^{\prime}$ of degree 8; for instance, this is always the case when $S^{\prime}$ is a quadric in $\mathbb{P}^{3}$, or when $\operatorname{rk} \operatorname{Pic}\left(S^{\prime}\right)=1$, cf. [ShV18, Lemma 7.3].

The next assertion follows from the classification of Sarkisov links of type IV, see [196, Theorem 2.6].

Lemma 2.8. Let $S$ be a del Pezzo surface of degree different from 1, 2, 4, and 8 over a field of characteristic zero such that $\operatorname{rk} \operatorname{Pic}(S)=2$. Suppose that one of the two extremal contractions from $S$ is a conic bundle. Then the other extremal contraction is not a conic bundle.

Now we can describe possible birational models of cubic surfaces without points. 
Lemma 2.9 (cf. Sh20a, Corollary 2.5]). Let $S$ be a smooth cubic surface over a field $\mathbb{K}$ of characteristic zero with $S(\mathbb{K})=\varnothing$. The following assertions hold.

(i) One has $\operatorname{rk} \operatorname{Pic}(S) \leqslant 3$.

(ii) If $\operatorname{rk} \operatorname{Pic}(S)=3$, then $S$ is birational to a non-trivial Severi-Brauer surface.

(iii) If $\operatorname{rk} \operatorname{Pic}(S)=2$, then $S$ is birational either to a non-trivial Severi-Brauer surface, or to a del Pezzo surface $S^{\prime}$ of degree 6 with $\operatorname{rk} \operatorname{Pic}\left(S^{\prime}\right)=1$.

Proof. Suppose that $\operatorname{rk} \operatorname{Pic}(S) \geqslant 2$. If $\operatorname{rk} \operatorname{Pic}(S) \geqslant 3$, then there is an extremal contraction $S \rightarrow S^{\prime}$ to a smooth surface $S^{\prime}$. If $\operatorname{rk} \operatorname{Pic}(S)=2$, then there also exists an extremal contraction $S \rightarrow S^{\prime}$ to a smooth surface $S^{\prime}$ by Lemma 2.8. In both cases $S^{\prime}$ is a a del Pezzo surface of degree $d^{\prime}>3$ with

$$
\operatorname{rk} \operatorname{Pic}\left(S^{\prime}\right)=\operatorname{rk} \operatorname{Pic}(S)-1 \text {. }
$$

Note that $S^{\prime}$ has no $\mathbb{K}$-points by Theorem 2.1. Furthermore, by Lemmas 2.4, 2.5, and 2.6, one has $d^{\prime}=6$ or $d^{\prime}=9$. In particular, this proves assertion (iii).

Now suppose that $\operatorname{rk} \operatorname{Pic}(S) \geqslant 3$. Then $\operatorname{rk} \operatorname{Pic}\left(S^{\prime}\right) \geqslant 2$, and hence $d^{\prime}$ cannot be equal to 9; so, we have $d^{\prime}=6$. As before, it follows from Lemma 2.8 that there exists an extremal contraction $S^{\prime} \rightarrow S^{\prime \prime}$ to a del Pezzo surface $S^{\prime \prime}$ of degree $d^{\prime \prime}>6$ with

$$
\operatorname{rk} \operatorname{Pic}\left(S^{\prime \prime}\right)=\operatorname{rk} \operatorname{Pic}\left(S^{\prime}\right)-1 .
$$

Applying Lemmas 2.5] and 2.6, we conclude that $d^{\prime \prime}$ cannot be equal to 7 and 8 . Therefore, one has $d^{\prime \prime}=9$, so that $S^{\prime \prime}$ is a Severi-Brauer surface. In particular, one has $\operatorname{rk} \operatorname{Pic}\left(S^{\prime \prime}\right)=1$, and hence $\operatorname{rk} \operatorname{Pic}(S)=3$. This proves assertions (i) and (ii).

We will complete a classification of birational models of cubic surfaces without points in Section 6, where we prove Proposition 1.5.

For the next result we refer the reader to [Ma86, Theorem V.5.1] and [Ch05, Theorem 1.5.6]; it can be also deduced from [I96, Theorem 2.6].

Theorem 2.10. Let $S$ be a smooth cubic surface over a field $\mathbb{K}$ of characteristic zero such that $\operatorname{rk} \operatorname{Pic}(S)=1$. Let $S^{\prime}$ be a smooth surface over $\mathbb{K}$ such that $S^{\prime}$ is either a del Pezzo surface with $\operatorname{rk} \operatorname{Pic}\left(S^{\prime}\right)=1$, or a conic bundle with $\operatorname{rk} \operatorname{Pic}\left(S^{\prime}\right)=2$. Suppose that $S^{\prime}$ is birational to $S$. Then $S^{\prime} \cong S$. Moreover, if $S(\mathbb{K})=\varnothing$, then $\operatorname{Bir}(S)=\operatorname{Aut}(S)$.

Corollary 2.11. Let $S$ be a smooth cubic surface over a field $\mathbb{K}$ of characteristic zero such that $S(\mathbb{K})=\varnothing$ and $\mathrm{rk} \operatorname{Pic}(S)=1$. Let $S^{\prime}$ be a smooth surface over $\mathbb{K}$ such that $S^{\prime}$ is either a del Pezzo surface or a conic bundle. Suppose that $S^{\prime}$ is birational to $S$. Then $S^{\prime} \cong S$. In particular, $S^{\prime}$ is not a conic bundle.

Proof. If there is a conic bundle structure $S^{\prime} \rightarrow C$, we can perform extremal contractions over $C$ if necessary and assume that $\operatorname{rk} \operatorname{Pic}\left(S^{\prime}\right)=2$; this is impossible by Theorem 2.10 , Therefore, we will assume that $S^{\prime}$ is a del Pezzo surface. Let $d^{\prime}$ be the degree of $S^{\prime}$. Note that $d^{\prime} \geqslant 3$ by Corollary 2.3. Suppose that either $d^{\prime}>3$, or $d^{\prime}=3$ and $\operatorname{rk} \operatorname{Pic}\left(S^{\prime}\right)>1$. We can perform several extremal contractions if necessary and obtain a del Pezzo surface $S^{\prime \prime}$ of degree $d^{\prime \prime}>3$ and $\operatorname{rk} \operatorname{Pic}\left(S^{\prime \prime}\right)=1$. The surface $S^{\prime \prime}$ is birational to $S$, which is again impossible by Theorem 2.10 .

The following result is a partial generalization of Theorem 2.10 to the equivariant setting; it is well known to experts. 
Lemma 2.12. Let $S$ be a smooth cubic surface over a field $\mathbb{K}$ of characteristic zero with $S(\mathbb{K})=\varnothing$. Let $G$ be a group acting on $S$ such that $\operatorname{rk} \operatorname{Pic}(S)^{G}=1$. Let $S^{\prime}$ be a del Pezzo surface over $\mathbb{K}$ with $\operatorname{rk} \operatorname{Pic}\left(S^{\prime}\right)^{G}=1$, and let $\xi: S \rightarrow S^{\prime}$ be a G-equivariant birational map. Then $\xi$ is an isomorphism.

Proof. Suppose that $\xi$ is not an isomorphism. Choose a very ample $G$-invariant linear system $\mathscr{L}^{\prime}$ on $S^{\prime}$, and let $\mathscr{L}$ be its proper transform on $S$. Then $\mathscr{L}$ is a mobile non-empty $G$-invariant linear system on $S$. Since $\operatorname{rk} \operatorname{Pic}(S)^{G}=1$, we can write

$$
\mathscr{L} \sim_{\mathbb{Q}}-\theta K_{S}
$$

for some positive rational number $\theta$. It follows from the Noether-Fano inequalities (see [196, Lemma 2.4(ii)]) that one has $\operatorname{mult}_{P}(\mathscr{L})>\theta$ for some point $P$ on $S$. Let $r$ be the degree of $P$, and let $L_{1}$ and $L_{2}$ be two general members of the linear system $\mathscr{L}$ (defined over $\overline{\mathbb{K}})$. We see that

$$
3 \theta^{2}=L_{1} \cdot L_{2} \geqslant r \operatorname{mult}_{P}(\mathscr{L})^{2}>r \theta^{2}
$$

and thus $r \leqslant 2$. On the other hand, $S$ has no points of degree 1 and 2 over $\mathbb{K}$ by Lemma 2.2. The obtained contradiction shows that $\xi$ is an isomorphism.

\section{MAIN EXAMPLE}

In this section we provide a construction that proves Theorem 1.2 ,

Let $\mathbb{k}$ be an algebraically closed field of characteristic zero, and let $\mathbb{K}=\mathbb{k}(\lambda, \mu)$, where $\lambda$ and $\mu$ are transcendental variables. Consider the cubic surface $S$ over $\mathbb{K}$ given in the projective space $\mathbb{P}^{3}$ with homogeneous coordinates $x, y, z$, and $t$ by the equation

$$
\lambda x^{3}+\lambda^{2} y^{3}+\mu z^{3}+\mu^{2} t^{3}=0 .
$$

It is straightforward to check that $S$ has no $\mathbb{K}$-points. Let $\omega$ be a non-trivial cubic root of unity. Consider the linear transformations

$$
\begin{aligned}
& \sigma_{1}:(x: y: z: t) \mapsto(\omega x: y: z: t), \\
& \sigma_{2}:(x: y: z: t) \mapsto(x: \omega y: z: t), \\
& \sigma_{3}:(x: y: z: t) \mapsto(x: y: \omega z: t) .
\end{aligned}
$$

Then $\sigma_{1}, \sigma_{2}$, and $\sigma_{3}$ generate a subgroup $G$ of $\operatorname{Aut}(S)$ isomorphic to $\boldsymbol{\mu}_{3}^{3}$.

Recall that the action of the groups $\operatorname{Aut}(S)$ and $\mathrm{Gal}(\overline{\mathbb{K}} / \mathbb{K})$ on $(-1)$-curves on $S^{\prime}$ defines homomorphisms of these groups to the Weyl group $\mathrm{W}\left(\mathrm{E}_{6}\right)$. Furthermore, the homomorphism $\operatorname{Aut}(S) \rightarrow \mathrm{W}\left(\mathrm{E}_{6}\right)$ is an embedding, see [Do12, Corollary 8.2.40]. Thus we can identify the group $\operatorname{Aut}(S)$ with its image in $\mathrm{W}\left(\mathrm{E}_{6}\right)$. In the notation of [Ca72], the elements $\sigma_{i}, 1 \leqslant i \leqslant 3$, have type $\mathrm{A}_{2}^{3}$; this follows from the fact that the quotient of $S_{\overline{\mathbb{K}}}$ by the group $G_{i} \cong \boldsymbol{\mu}_{3}$ generated by the element $\sigma_{i}$ is isomorphic to $\mathbb{P}^{2}$, and thus $\operatorname{rk} \operatorname{Pic}\left(S_{\overline{\mathbb{K}}}\right)^{G_{i}}=1$.

Let $\gamma_{\lambda}$ and $\gamma_{\mu}$ be the images in $\mathrm{W}\left(\mathrm{E}_{6}\right)$ of generators of the Galois groups $\operatorname{Gal}(\mathbb{K}(\sqrt[3]{\lambda}) / \mathbb{K})$ and $\operatorname{Gal}(\mathbb{K}(\sqrt[3]{\mu}) / \mathbb{K})$. Then $\gamma_{\lambda}$ and $\gamma_{\mu}$ are elements of order 3 
that commute with each other. Furthermore, the 27 lines on $S_{\overline{\mathbb{K}}}$ are given by equations

$$
\begin{array}{r}
\sqrt[3]{\lambda} x+\omega^{i} \sqrt[3]{\lambda^{2}} y=\sqrt[3]{\mu} z+\omega^{j} \sqrt[3]{\mu^{2}} t=0 \\
\sqrt[3]{\lambda} x+\omega^{i} \sqrt[3]{\mu} z=\sqrt[3]{\lambda^{2}} y+\omega^{j} \sqrt[3]{\mu^{2}} t=0 \\
\sqrt[3]{\lambda} x+\omega^{i} \sqrt[3]{\mu^{2}} t=\sqrt[3]{\lambda^{2}} y+\omega^{j} \sqrt[3]{\mu} z=0 \\
i, j \in\{0,1,2\}
\end{array}
$$

Thus each of the 27 lines on $S_{\overline{\mathbb{K}}}$ is defined over the field $\mathbb{K}(\sqrt[3]{\lambda}, \sqrt[3]{\mu})$. Hence the image of the whole Galois group $\mathrm{Gal}(\overline{\mathbb{K}} / \mathbb{K})$ in the Weyl group $\mathrm{W}\left(\mathrm{E}_{6}\right)$ is the group $\Gamma \cong \boldsymbol{\mu}_{3}^{2}$ generated by $\gamma_{\lambda}$ and $\gamma_{\mu}$.

Note that the group $\Gamma$ can be generated by the elements

$$
\gamma_{1}=\gamma_{\lambda} \gamma_{\mu} \text { and } \gamma_{2}=\gamma_{\lambda} \gamma_{\mu}^{-1}
$$

The element $\gamma_{1}$ has nine invariant lines on $S_{\overline{\mathbb{K}}}$, and the remaining 18 lines split into a union of six $\gamma_{1}$-invariant triples of pairwise disjoint lines. This means that $\gamma_{1}$ has type $A_{2}$ in the notation of [Ca72]; indeed, the other options for an element of order 3 are types $\mathrm{A}_{2}^{2}$ and $\mathrm{A}_{2}^{3}$, but elements of any of these two types have no invariant lines on $S_{\overline{\mathbb{K}}}$. Similarly, the element $\gamma_{2}$ also has type $\mathrm{A}_{2}$. Therefore, for $i=1,2$ the subspace $V_{i}$ of the $\mathbb{C}$-vector space

$$
V=\operatorname{Pic}\left(S_{\overline{\mathbb{K}}}\right) \otimes \mathbb{C}
$$

spanned by the eigen-vectors of $\gamma_{i}$ with non-trivial eigen-values has dimension 2. Denote by $\left\langle V_{1}, V_{2}\right\rangle$ the subspace in $V$ generated by $V_{1}$ and $V_{2}$. Since $\Gamma$ is an abelian group generated by $\gamma_{\lambda}$ and $\gamma_{\mu}$, we can bound the rank of the $\Gamma$-invariant sublattice $\operatorname{Pic}\left(S_{\overline{\mathbb{K}}}\right)^{\Gamma}$ of $\operatorname{Pic}\left(S_{\overline{\mathbb{K}}}\right)$ as

$$
\operatorname{rk} \operatorname{Pic}\left(S_{\overline{\mathbb{K}}}\right)^{\Gamma}=\operatorname{dim} V-\operatorname{dim}\left\langle V_{1}, V_{2}\right\rangle \geqslant \operatorname{dim} V-\operatorname{dim} V_{1}-\operatorname{dim} V_{2}=7-2-2=3,
$$

so that

$$
\operatorname{rk} \operatorname{Pic}(S)=\operatorname{rk} \operatorname{Pic}\left(S_{\overline{\mathbb{K}}}\right)^{\Gamma} \geqslant 3
$$

In particular, $S$ is birational to some non-trivial Severi-Brauer surface $S_{0}$ by Lemma 2.9(i),(ii), and $G$ is embedded as a subgroup into $\operatorname{Bir}\left(S_{0}\right)$.

Let $G^{\prime}$ denote the subgroup of $G$ generated by $\sigma_{3}$, and let $G^{\prime \prime}$ denote the subgroup generated by $\sigma_{2}$ and $\sigma_{3}$, so that $G^{\prime} \cong \boldsymbol{\mu}_{3}$ and $G^{\prime \prime} \cong \boldsymbol{\mu}_{3}^{2}$. Since the element $\sigma_{3}$ has type $\mathrm{A}_{2}^{3}$, one has

$$
\operatorname{rk} \operatorname{Pic}(S)^{G^{\prime}}=1 .
$$

According to Lemma 2.12, this implies there is no $G^{\prime}$-equivariant birational map from $S$ to $S_{0}$. Therefore, $G^{\prime}$ (and thus also $G^{\prime \prime}$ and $G$ ) is not conjugate in $\operatorname{Bir}\left(S_{0}\right)$ to a subgroup of $\operatorname{Aut}\left(S_{0}\right)$. This completes the proof of Theorem 1.2.

\section{Plane Cubics}

In this section we discuss the action of the automorphism group of a plane cubic curve without points over the base field on the inflection points of the curve.

Recall that every smooth plane cubic curve over a field $\mathbb{K}$ of characteristic zero has exactly 9 inflection points over $\overline{\mathbb{K}}$. The set $\Sigma \subset \mathbb{P}^{2}$ of these points is defined over $\mathbb{K}$. On the other hand, the set $\Sigma_{\overline{\mathbb{K}}}$ together with the lines passing through its points forms a configuration $\left(9_{4}, 12_{3}\right)$ isomorphic to the configuration of points and lines on the affine 
plane $\mathbb{A}^{2}$ over the field $\mathbf{F}_{3}$ of three elements. Thus, the automorphism group of the configuration $\Sigma_{\overline{\mathbb{K}}}$ is isomorphic to the group

$$
\mathrm{GA}_{2}\left(\mathbf{F}_{3}\right) \cong \boldsymbol{\mu}_{3}^{2} \rtimes \mathrm{GL}_{2}\left(\mathbf{F}_{3}\right)
$$

of order $432=16 \cdot 27$; we refer the reader to [BK86, $\S 7.3]$ for more details.

Lemma 4.1. Let $H \subset \mathrm{GA}_{2}\left(\mathbf{F}_{3}\right)$ be a subgroup acting on $\mathbb{A}^{2}\left(\mathbf{F}_{3}\right)$ without fixed points. Then $H$ contains an element of order 3.

Proof. Suppose that $H$ has no elements of order 3. Then the order of $H$ is a power of 2 . Since $H$ has no fixed points on $\mathbb{A}^{2}\left(\mathbf{F}_{3}\right)$, we conclude that $\mathbb{A}^{2}\left(\mathbf{F}_{3}\right)$ is a disjoint union of $H$-orbits of even order. This is impossible because $\left|\mathbb{A}^{2}\left(\mathbf{F}_{3}\right)\right|=9$.

Corollary 4.2. Let $C$ be a smooth plane cubic curve over a field $\mathbb{K}$ of characteristic zero, and let $\Sigma \subset \mathbb{P}^{2}$ be the set of its inflection points. Suppose that $\Sigma(\mathbb{K})=\varnothing$. Then the image of the Galois group $\mathrm{Gal}(\overline{\mathbb{K}} / \mathbb{K})$ in the group $\mathrm{GA}_{2}\left(\mathbf{F}_{3}\right) \cong \operatorname{Aut}\left(\Sigma_{\overline{\mathbb{K}}}\right)$ contains an element of order 3 .

\section{Automorphism Groups}

In this section we prove the first assertion of Theorem 1.4.

Lemma 5.1. Let $S$ be a smooth cubic surface over a field $\mathbb{K}$ of characteristic zero with $S(\mathbb{K})=\varnothing$. Then the order of the group $\operatorname{Aut}(S)$ is odd.

Proof. The argument is identical to the one used in the proof of [Sh20a, Lemma 3.3]. Suppose that the order of $\operatorname{Aut}(S)$ is even. Then there exists an automorphism $g$ of $S$ of order 2. The action of $g$ on $S_{\overline{\mathbb{K}}}$ can be of one of the two types listed in [T16, Table 2]; in the notation of [T16, Table 2] these are types 1 and 2. If $g$ is of type 1, then the fixed point locus $\operatorname{Fix}_{S_{\overline{\mathrm{K}}}}(g)$ of $g$ on $S_{\overline{\mathbb{K}}}$ consists of a smooth elliptic curve and one isolated point; if $g$ is of type 2, then $\operatorname{Fix}_{S_{\overline{\mathbb{K}}}}(g)$ consists of a $(-1)$-curve and three isolated points. Note that a $\operatorname{Gal}(\overline{\mathbb{K}} / \mathbb{K})$-invariant $(-1)$-curve on $S$ always contains a $\mathbb{K}$-point, since such a curve is a line in the anticanonical embedding of $S$. Therefore, in each of these two cases we find a $\mathrm{Gal}(\overline{\mathbb{K}} / \mathbb{K})$-invariant point on $S_{\overline{\mathbb{K}}}$, which gives a contradiction.

Knowing that a group acting on a cubic surface has odd order provides strong restrictions on its structure. In particular, Lemma 5.1 implies the following.

Corollary 5.2. Let $S$ be a smooth cubic surface over a field $\mathbb{K}$ of characteristic zero with $S(\mathbb{K})=\varnothing$. Suppose that $S_{\overline{\mathbb{K}}}$ is of one of the types II, V, VI, or VIII in the notation of [Do12, Table 9.6]. Then the group $\operatorname{Aut}(S)$ is cyclic.

Proof. According to [Do12, Table 9.6], one has

$$
\operatorname{Aut}\left(S_{\overline{\mathbb{K}}}\right) \subset \mathfrak{S}_{5} .
$$

On the other hand, the order of $\operatorname{Aut}(S)$ is odd by Lemma 5.1, and thus $\operatorname{Aut}(S)$ is a cyclic group of order either 3 or 5 (note that $\mathfrak{S}_{5}$ does not contain groups of order 15 ).

Let $\mathscr{H}_{3}$ denote the Heisenberg group of order 27; this is the only non-abelian group of order 27 and exponent 3 . Its center $\mathrm{z}\left(\mathscr{H}_{3}\right)$ is isomorphic to $\boldsymbol{\mu}_{3}$, and there is a non-split exact sequence

$$
1 \rightarrow \mathrm{z}\left(\mathscr{H}_{3}\right) \rightarrow \underset{8}{\mathscr{H}_{3}} \rightarrow \boldsymbol{\mu}_{3}^{2} \rightarrow 1
$$


On the other hand, there is an isomorphism

$$
\mathscr{H}_{3} \cong \boldsymbol{\mu}_{3}^{2} \rtimes \boldsymbol{\mu}_{3} .
$$

Lemma 5.3. Let $S$ be a smooth cubic surface over a field $\mathbb{K}$ of characteristic zero with $S(\mathbb{K})=\varnothing$. Suppose that $S_{\overline{\mathbb{K}}}$ is of one of the types I, III, or IV in the notation of [Do12, Table 9.6]. Then the group $\operatorname{Aut}(S)$ is abelian.

Proof. The order of $\operatorname{Aut}(S)$ is odd by Lemma 5.1. Therefore, according to [Do12, Table 9.6], the group $\operatorname{Aut}(S)$ is a subgroup of the 3-Sylow subgroup $\Theta \subset \operatorname{Aut}\left(S_{\overline{\mathbb{K}}}\right)$; one has $\Theta \cong \boldsymbol{\mu}_{3}^{3} \rtimes \boldsymbol{\mu}_{3}$ if $S_{\overline{\mathbb{K}}}$ is of type I, and $\Theta \cong \mathscr{H}_{3}$ if $S_{\overline{\mathbb{K}}}$ is of type III or IV. In either case the central subgroup $\Xi$ of $\Theta$ is isomorphic to $\boldsymbol{\mu}_{3}$. Note that any subgroup of $\Theta$ that does not contain $\Xi$ is abelian. Thus we may assume that $\operatorname{Aut}(S)$ contains $\Xi$. Then $S / \Xi \cong \mathbb{P}^{2}$, and the quotient map

$$
\nu: S \rightarrow \mathbb{P}^{2}
$$

is defined over $\mathbb{K}$. The branch curve of $\nu$ is a smooth plane cubic $C$. Since $S$ has no $\mathbb{K}$-points, the curve $C$ also has no $\mathbb{K}$-points. Therefore, the image of the Galois group $\mathrm{Gal}(\overline{\mathbb{K}} / \mathbb{K})$ in the automorphism group $\mathrm{GA}_{2}\left(\mathbf{F}_{3}\right)$ of the configuration of the inflection points of $C_{\overline{\mathbb{K}}}$ contains an element of order 3 by Corollary 4.2. Since the map $\nu$ is $\operatorname{Gal}(\overline{\mathbb{K}} / \mathbb{K})$-equivariant, we see that the image of Gal $(\overline{\mathbb{K}} / \mathbb{K})$ in the Weyl group $W\left(\mathrm{E}_{6}\right)$ contains an element $\gamma$ of order 3 such that $\gamma$ is not contained in the image of the group $\Xi$ in $\mathrm{W}\left(\mathrm{E}_{6}\right)$. On the other hand, we know that the group $\operatorname{Aut}(S)$ must commute with $\gamma$. Recall that the 3 -Sylow subgroup $\Delta$ of $\mathrm{W}\left(\mathrm{E}_{6}\right)$ is isomorphic to $\boldsymbol{\mu}_{3}^{3} \rtimes \boldsymbol{\mu}_{3}$. The center of $\Delta$ is isomorphic to $\boldsymbol{\mu}_{3}$, and according to our assumptions it coincides with the image in the Weyl group $\mathrm{W}\left(\mathrm{E}_{6}\right)$ of the subgroup $\Xi \subset \operatorname{Aut}(S)$. Therefore, $\operatorname{Aut}(S)$ is isomorphic to a subgroup of $\Delta$ commuting with a non-central element $\gamma \in \Delta$. It is straightforward to check that any such subgroup of $\Delta$ is abelian.

The following example shows that in the notation of the proof of Lemma 5.3 the subgroup in $\operatorname{Aut}\left(\mathbb{P}^{2}\right)$ preserving the plane cubic $C$ may be non-abelian, although the group $\operatorname{Aut}(S)$ is abelian.

Example 5.4. Let $\mathbb{k}$ be an algebraically closed field of characteristic zero, and let $\mathbb{K}=\mathbb{k}(\lambda, \mu)$, where $\lambda$ and $\mu$ are transcendental variables. Consider the cubic surface $S$ over $\mathbb{K}$ given by the equation

$$
\mu t^{3}=x^{3}+\lambda y^{3}+\lambda^{2} z^{3}
$$

in the notation of [Do12, Table 9.6], the surface $S_{\overline{\mathbb{K}}}$ has type I. It is easy to see that $S$ has no $\mathbb{K}$-points. Let $\omega$ be a non-trivial cubic root of unity. Let $\sigma_{1}, \sigma_{2}$, and $\sigma_{3}$ be the automorphisms of $S$ defined as

$$
\begin{aligned}
& \sigma_{1}:(x: y: z: t) \mapsto(\omega x: y: z: t), \\
& \sigma_{2}:(x: y: z: t) \mapsto(x: \omega y: z: t), \\
& \sigma_{3}:(x: y: z: t) \mapsto(x: y: z: \omega t) .
\end{aligned}
$$

Let $\nu: S \rightarrow \mathbb{P}^{2}$ be the quotient by the group generated by $\sigma_{3}$. Denote by $C$ the branch curve of $\nu$. Then $C$ is a smooth plane cubic given in $\mathbb{P}^{2}$ with homogeneous coordinates $x, y$, and $z$ by the equation

$$
x^{3}+\lambda y^{3}+\lambda^{2} z^{3}=0 .
$$


Let $\omega$ be a non-trivial cubic root of unity. The group $G \cong \boldsymbol{\mu}_{3}^{3}$ generated by $\sigma_{1}, \sigma_{2}$, and $\sigma_{3}$ acts on $S$, and its quotient $\hat{G} \cong \boldsymbol{\mu}_{3}^{2}$ generated by the linear transformations

$$
\begin{aligned}
& \hat{\sigma}_{1}:(x: y: z) \mapsto(\omega x: y: z), \\
& \hat{\sigma}_{2}:(x: y: z) \mapsto(x: \omega y: z),
\end{aligned}
$$

acts on $\mathbb{P}^{2}$ so that the map $\nu$ is $G$-equivariant. The group $\hat{G}$ preserves the plane curve $C$, but $C$ actually has more symmetries. Namely, it is also preserved by the linear transformation

$$
\hat{\sigma}:(x: y: z) \mapsto(\lambda z: x: y)
$$

Note that $\hat{\sigma}$ lifts to the automorphism

$$
\sigma:(x: y: z: t) \mapsto(\lambda z: x: y: \sqrt[3]{\lambda} t)
$$

of the surface $S_{\overline{\mathbb{K}}}$. However, we can see from Lemma 5.3 (or check in a straightforward way) that $\hat{\sigma}$ cannot be lifted to an automorphism of $S$.

Now we are ready to prove assertion (i) of Theorem 1.4.

Lemma 5.5. Let $\mathbb{K}$ be a field of characteristic zero, and let $S$ be a smooth cubic surface over $\mathbb{K}$. Suppose that $S$ has no $\mathbb{K}$-points. Then the group $\operatorname{Aut}(S)$ is abelian.

Proof. Inspecting [Do12, Table 9.6] which lists all possible automorphism groups of smooth cubic surfaces over fields of characteristic zero, we see that the group $\operatorname{Aut}\left(S_{\overline{\mathbb{K}}}\right)$ is abelian if $S_{\overline{\mathbb{K}}}$ is of one of the types VII, IX, X, or XI; the $\operatorname{group} \operatorname{Aut}(S)$ is cyclic by Corollary 5.2, if $S_{\overline{\mathbb{K}}}$ is of one of the types II, V, VI, or VIII; and $\operatorname{Aut}(S)$ is abelian by Lemma 5.3, if $S_{\overline{\mathbb{K}}}$ is of one of the types I, III, or IV.

Remark 5.6. If $S$ is a smooth cubic surface over a field $\mathbb{K}$ of characteristic zero such that $S_{\overline{\mathbb{K}}}$ is of one of the types VII, IX, X, or XI, then $S$ has a $\mathbb{K}$-point. Indeed, if $S_{\overline{\mathbb{K}}}$ is of one of the types VII, IX, or XI, then $S_{\overline{\mathbb{K}}}$ has a unique Eckardt point, see [Do12, Table 9.6]; note that there is a typo for type VII in [Do12, Table 9.6], cf. the relation between Eckardt points and certain involutions of cubics described in [Do12, Proposition 9.1.23]. Thus $S$ has a $\mathbb{K}$-point. If $S_{\overline{\mathbb{K}}}$ is of type X, then $S_{\overline{\mathbb{K}}}$ has exactly two Eckardt points, and thus $S$ has a $\mathbb{K}$-point by Lemma 2.2 . This means that these cases do not actually arise in the proof of Lemma 5.5.

\section{Del Pezzo surfaces of Degree 6}

In this section we make some observations on del Pezzo surfaces of degree 6.

Lemma 6.1. Let $S$ be a del Pezzo surface of degree 6 over a field $\mathbb{K}$ of characteristic zero such that $S(\mathbb{K})=\varnothing$ and $\operatorname{rk} \operatorname{Pic}(S)=1$. Let $S^{\prime}$ be a smooth surface over $\mathbb{K}$ such that $S^{\prime}$ is either a del Pezzo surface with $\operatorname{rkPic}\left(S^{\prime}\right)=1$, or a conic bundle with $\operatorname{rk} \operatorname{Pic}\left(S^{\prime}\right)=2$. Suppose that $S^{\prime}$ is birational to $S$. Then $S^{\prime}$ is a del Pezzo surface of degree 6 with $\operatorname{rk} \operatorname{Pic}\left(S^{\prime}\right)=1$.

Proof. Choose a birational map $S \rightarrow S^{\prime}$, and decompose it into a sequence of Sarkisov links

$$
S_{--\rightarrow}^{\chi_{1}} S_{1} \stackrel{\chi_{2}}{-\rightarrow} \ldots \stackrel{\chi_{n}}{\rightarrow} S_{n}=S^{\prime} .
$$

We know from [196, Theorem 2.6] that $S_{1}$ is again a del Pezzo surface of degree 6 with $\operatorname{rk} \operatorname{Pic}\left(S_{1}\right)=1$. Moreover, one has $S_{i}(\mathbb{K})=\varnothing$ by Theorem 2.1. Thus, the required assertion follows by induction on $n$. 
Corollary 6.2. Let $S$ be a del Pezzo surface of degree 6 over a field $\mathbb{K}$ of characteristic zero such that $\operatorname{rk} \operatorname{Pic}(S)=1$. Suppose that $S$ has no points of degree 1 and 2 over $\mathbb{K}$. Let $S^{\prime}$ be a smooth surface over $\mathbb{K}$ such that $S^{\prime}$ is either a del Pezzo surface or a conic bundle. Suppose that $S^{\prime}$ is birational to $S$. Then $S^{\prime}$ is a del Pezzo surface of degree 3 or 6 ; in the latter case one has $\mathrm{rk} \operatorname{Pic}\left(S^{\prime}\right)=1$. Furthermore, $S^{\prime}$ is not a conic bundle.

Proof. If there is a conic bundle structure $S^{\prime} \rightarrow C$, we can perform extremal contractions over $C$ if necessary and assume that $\operatorname{rk} \operatorname{Pic}\left(S^{\prime}\right)=2$; this is impossible by Lemma 6.1. Therefore, we will assume that $S^{\prime}$ is a del Pezzo surface. Let $d^{\prime}$ be the degree of $S^{\prime}$. Note that $S^{\prime}$ does not contain points of degree 1 and 2 over $\mathbb{K}$ by Theorem 2.1 , In particular, one has $d^{\prime}>2$, because $S^{\prime}$ always has a $\mathbb{K}$-point of degree at most $d^{\prime}$. Similarly, $d^{\prime} \neq 5$ because a del Pezzo surface of degree 5 always has a $\mathbb{K}$-point.

Suppose that either $d^{\prime}>6$, or $d^{\prime}=6$ and $\operatorname{rk} \operatorname{Pic}\left(S^{\prime}\right)>1$. We can perform several extremal contractions if necessary and obtain a del Pezzo surface $S^{\prime \prime}$ of degree $d^{\prime \prime}>6$ and $\operatorname{rk} \operatorname{Pic}\left(S^{\prime \prime}\right)=1$. The surface $S^{\prime \prime}$ is birational to $S$, which is impossible by Lemma 6.1,

Now suppose that $d^{\prime}=4$. By Lemma 6.1 one has rk $\operatorname{Pic}\left(S^{\prime}\right)>1$. Performing several extremal contractions, we obtain a del Pezzo surface $S^{\prime \prime}$ of degree $d^{\prime \prime}>d^{\prime}$ and $\operatorname{rk} \operatorname{Pic}\left(S^{\prime \prime}\right)=1$. Again applying Lemma 6.1, we see that $d^{\prime \prime}=6$. Thus the morphism $S^{\prime} \rightarrow S^{\prime \prime}$ contracts two $(-1)$-curves over $\overline{\mathbb{K}}$, and so $S^{\prime \prime}$ contains a $\mathbb{K}$-point of degree at most 2 over $\mathbb{K}$. This is impossible by Theorem 2.1 ,

Therefore, the only possible cases are $d^{\prime}=3$ and $d^{\prime}=6$, and in the latter case one has $\operatorname{rk} \operatorname{Pic}\left(S^{\prime}\right)=1$.

Let $S$ be a del Pezzo surface of degree 6 over a field $\mathbb{K}$ of characteristic zero. Recall from [Do12, Theorem 8.4.2] that

$$
\operatorname{Aut}\left(S_{\overline{\mathbb{K}}}\right) \cong\left(\overline{\mathbb{K}}^{*}\right)^{2} \rtimes\left(\mathfrak{S}_{3} \times \boldsymbol{\mu}_{2}\right) .
$$

The configuration of $(-1)$-curves on $S_{\overline{\mathbb{K}}}$ can be interpreted as a hexagon, and the group $\mathfrak{S}_{3} \times \boldsymbol{\mu}_{2}$ on the right hand side of (6.3) can be identified with the automorphism group of this configuration. Note that there are two possible ways to choose the factor $\mathfrak{S}_{3}$ in $\mathfrak{S}_{3} \times \boldsymbol{\mu}_{2}$; we will always assume that this subgroup is chosen so that it preserves the two triples of pairwise disjoint $(-1)$-curves.

Lemma 6.4. Let $S$ be a del Pezzo surface of degree 6 over a field $\mathbb{K}$ of characteristic zero. Suppose that $S$ has no points of degree 1 and 2 over $\mathbb{K}$. Then the image of the group $\operatorname{Aut}(S)$ in $\mathfrak{S}_{3} \times \boldsymbol{\mu}_{2}$ does not contain the central element $z$ of $\mathfrak{S}_{3} \times \boldsymbol{\mu}_{2}$.

Proof. Suppose that the image of $\operatorname{Aut}(S)$ contains $z$. Let $\tilde{z}$ be an element of $\operatorname{Aut}(S)$ mapped to $z$.

There exists a quadratic extension $\mathbb{L} / \mathbb{K}$ such that the image of the Galois group Gal $(\overline{\mathbb{K}} / \mathbb{L})$ in $\mathfrak{S}_{3} \times \boldsymbol{\mu}_{2}$ is contained in the factor $\mathfrak{S}_{3}$ of $\mathfrak{S}_{3} \times \boldsymbol{\mu}_{2}$. Thus Gal $(\overline{\mathbb{K}} / \mathbb{L})$ preserves the two triples of pairwise disjoint $(-1)$-curves on $S_{\overline{\mathbb{K}}}$. This means that the surface $S_{\mathbb{L}}$ has two contractions

$$
\pi_{i}: S_{\mathbb{L}} \rightarrow B_{i}, \quad i=1,2,
$$

where $B_{i}$ are Severi-Brauer surfaces over $\mathbb{L}$. Consider the birational map

$$
\pi_{2} \circ \pi_{1}^{-1}: B_{1} \rightarrow B_{2} .
$$

After extension of scalars to $\overline{\mathbb{K}}$ it defines a birational map $\mathbb{P}^{2} \rightarrow-\rightarrow \mathbb{P}^{2}$ that is given by a certain linear system of conics (see [Sh20a, §2] and references therein). This implies 
that $B_{1}$ and $B_{2}$ correspond to opposite central simple algebras, see e.g. GSh18, Exercise 3.3.7(iii)]. On the other hand, the element $\tilde{z} \in \operatorname{Aut}\left(S_{\mathbb{L}}\right)$ swaps the contractions $\pi_{1}$ and $\pi_{2}$, which means that $B_{1} \cong B_{2}$. Therefore, both $B_{1}$ and $B_{2}$ are isomorphic to $\mathbb{P}^{2}$. In particular, the surface $S_{\mathbb{L}}$ has an $\mathbb{L}$-point. Hence $S$ has a $\mathbb{K}$-point of degree at most 2 , which contradicts our assumptions.

Lemma 6.5. Let $S$ be a del Pezzo surface of degree 6 over a field $\mathbb{K}$ of characteristic zero such that $\operatorname{rk} \operatorname{Pic}(S)=1$. Suppose that $S$ has no points of degree 1 and 2 over $\mathbb{K}$. Then the group $\operatorname{Aut}(S)$ has a normal abelian subgroup of index at most 3.

Proof. Let $\Gamma$ be the image of the group $\operatorname{Gal}(\overline{\mathbb{K}} / \mathbb{K})$ in $\mathfrak{S}_{3} \times \boldsymbol{\mu}_{2}$. Since $\operatorname{rk} \operatorname{Pic}(S)=1$, we conclude that $\Gamma$ contains an element $\gamma$ of order 3 . The image $\Delta$ of $\operatorname{Aut}(S)$ in $\mathfrak{S}_{3} \times \boldsymbol{\mu}_{2}$ commutes with $\Gamma$, and thus it is contained in the centralizer of $\gamma$, which is the group of order 6 generated by $\gamma$ and the central element $z$ of $\mathfrak{S}_{3} \times \boldsymbol{\mu}_{2}$. Also, we know from Lemma 6.4 that $\Delta$ does not contain the element $z$. Hence $\Delta$ is contained in the group of order 3 generated by $\gamma$, and so the kernel of the homomorphism $\operatorname{Aut}(S) \rightarrow \mathfrak{S}_{3} \times \boldsymbol{\mu}_{2}$ is an abelian group of index at most 3 in $\operatorname{Aut}(S)$.

Corollary 6.6. Let $S$ be a del Pezzo surface of degree 6 over a field $\mathbb{K}$ of characteristic zero such that $\operatorname{rk} \operatorname{Pic}(S)=1$. Suppose that $S$ has no points of degree 1 and 2 over $\mathbb{K}$. Let $G$ be a finite subgroup of $\operatorname{Bir}(S)$. Then $G$ has a normal abelian subgroup of index at most 3 .

Proof. Regularizing the action of $G$ and running a $G$-Minimal Model Program, we obtain a surface $S^{\prime}$ with an action of $G$ and a $G$-equivariant birational map $S \rightarrow S^{\prime}$, such that $S^{\prime}$ is either a del Pezzo surface, or a conic bundle. Note that $S^{\prime}$ has no points of degree 1 and 2 over $\mathbb{K}$ by Theorem [2.1. Applying Corollary 6.2, we see that $S^{\prime}$ is either a cubic surface, or a del Pezzo surface of degree 6 with $\operatorname{rk} \operatorname{Pic}\left(S^{\prime}\right)=1$. In the former case the group $G$ is abelian by Lemma 5.5. In the latter case the assertion follows from Lemma 6.5.

We conclude this section by a classification of birational models of cubic surfaces without points and del Pezzo surfaces of degree 6 without points of degree 1 and 2 over the base field. It is not necessary for the proof of the main results of this paper, but we find it interesting on its own.

Lemma 6.7 (cf. Lemma 2.9). Let $S$ be a del Pezzo surface of degree 6 over a field $\mathbb{K}$ of characteristic zero such that $S$ has no points of degree 1 and 2 over $\mathbb{K}$. The following assertions hold.

(i) One has $\operatorname{rk} \operatorname{Pic}(S) \leqslant 2$.

(ii) If $\operatorname{rk} \operatorname{Pic}(S)=2$, then $S$ is birational to a non-trivial Severi-Brauer surface.

Proof. Suppose that $\mathrm{rk} \operatorname{Pic}(S) \geqslant 2$. Keeping in mind Lemma 2.8, we see that there exists an extremal contraction $\pi: S \rightarrow S^{\prime}$ to a del Pezzo surface $S^{\prime}$ of degree $d^{\prime}>6$ with

$$
\operatorname{rk} \operatorname{Pic}\left(S^{\prime}\right)=\operatorname{rk} \operatorname{Pic}(S)-1 .
$$

The surface $S^{\prime}$ has no points of degree 1 and 2 over $\mathbb{K}$ by Theorem 2.1 . If $d^{\prime} \leqslant 8$, then $\pi$ contracts at most two $(-1)$-curves on $S_{\overline{\mathbb{K}}}$. This implies that $S^{\prime}$ has a $\mathbb{K}$-point of degree at most 2, which is a contradiction. Hence $d^{\prime}=9$, so that $S^{\prime}$ is a non-trivial Severi-Brauer surface. In particular, one has $\operatorname{rk} \operatorname{Pic}\left(S^{\prime}\right)=1$ and $\operatorname{rk} \operatorname{Pic}(S)=2$. 
We complete this section by a classification of birational models of smooth cubic surfaces without points and del Pezzo surfaces of degree 6 without points of degree 1 and 2 .

Proof of Proposition 1.5. Note that $S$ has no points of degree 1 and 2 over $\mathbb{K}$. If $S$ is a del Pezzo surface of degree 6, this holds by assumption; if $S$ is a cubic surface, then this holds by Lemma 2.2, if $S$ is a Severi-Brauer surface, this is well known, see e.g. Ko16, Theorem 53(2)]. Therefore, by Theorem 2.1 any surface $S^{\prime}$ birational to $S$ has no points of degree 1 and 2 over $\mathbb{K}$.

Suppose that $S$ is a non-trivial Severi-Brauer surface. Then the required assertion follows from [Sh20a, Corollary 2.4].

Suppose that $S$ is a del Pezzo surface of degree 6 with no points of degree 1 and 2 over $\mathbb{K}$. Then $\operatorname{rk} \operatorname{Pic}(S) \leqslant 2$ by Lemma 6.7(i). If $\operatorname{rk} \operatorname{Pic}(S)=1$, the assertion is given by Corollary 6.2. If $\operatorname{rk} \operatorname{Pic}(S)=2$, we know from Lemma 6.7(ii) that $S$ is birational to a Severi-Brauer surface, and the required assertion follows.

Finally, suppose that $S$ is a smooth cubic surface with $S(\mathbb{K})=\varnothing$. Then $\operatorname{rk} \operatorname{Pic}(S) \leqslant 3$ by Lemma 2.9(i). If $\operatorname{rk} \operatorname{Pic}(S)=1$, the assertion is given by Corollary 2.11, If $\operatorname{rk} \operatorname{Pic}(S) \geqslant 2$, we know from Lemma 2.9)(ii),(iii) that $S$ is birational either to a del Pezzo surface $S^{\prime}$ of degree 6 with $\operatorname{rk} \operatorname{Pic}\left(S^{\prime}\right)=1$, or to a Severi-Brauer surface. Therefore, in these cases the required assertion is implied by the above arguments.

\section{Birational AUtOMORPHism GROUPS}

In this section we prove the remaining assertions of Theorem 1.4. Let us start with deriving some consequences from Sections 2 and 6.

Corollary 7.1. Let $S$ be a smooth cubic surface over a field $\mathbb{K}$ of characteristic zero with $S(\mathbb{K})=\varnothing$ and $\mathrm{rk} \operatorname{Pic}(S)=3$. Then every finite subgroup of $\operatorname{Bir}(S)$ contains a normal abelian subgroup of index at most 3. Moreover, there exists a field $\mathbb{K}_{0}$ of characteristic zero, a smooth cubic surface $S_{0}$ over $\mathbb{K}_{0}$ with $S_{0}\left(\mathbb{K}_{0}\right)=\varnothing$ and $\operatorname{rk} \operatorname{Pic}\left(S_{0}\right)=3$, and a finite subgroup $G_{0} \subset \operatorname{Bir}\left(S_{0}\right)$ such that the minimal index of a normal abelian subgroup in $G_{0}$ equals 3.

Proof. We know from Lemma 2.9)(ii) that $S$ is birational to a non-trivial Severi-Brauer surface. Therefore, the first of the required assertions follows from Theorem 1.3.

To prove the second assertion, take a field $\mathbb{K}_{0}$ of characteristic zero and a non-trivial Severi-Brauer surface $S_{0}^{\prime}$ over $\mathbb{K}_{0}$ such that $\operatorname{Aut}\left(S_{0}^{\prime}\right)$ contains a non-abelian finite subgroup $G_{0}$; these exist by Theorem 1.3. Then the minimal index of a normal abelian subgroup in $G_{0}$ equals 3. Now we construct a cubic surface $S_{0}$ as a blow up of $S_{0}^{\prime}$ at two points of degree 3 over $\mathbb{K}_{0}$ in general position; note that such points always exist, see e.g. [Ko16, Theorem 53(3)]. Thus $S_{0}$ is a cubic surface without $\mathbb{K}_{0}$-points, and $\operatorname{Bir}\left(S_{0}\right)$ contains the group $G_{0}$.

Corollary 7.2. Let $S$ be a smooth cubic surface over a field $\mathbb{K}$ of characteristic zero with $S(\mathbb{K})=\varnothing$ and $\operatorname{rk} \operatorname{Pic}(S)=2$. Then every finite subgroup of $\operatorname{Bir}(S)$ contains an abelian subgroup of index at most 3 .

Proof. We know from Lemma 2.9)(iii) that $S$ is birational either to a non-trivial SeveriBrauer surface, or to a del Pezzo surface $S^{\prime}$ of degree 6 with $\operatorname{rk} \operatorname{Pic}\left(S^{\prime}\right)=1$. In the former case the assertion follows from Theorem 1.3. In the latter case we recall that $S$ does not contain points of degree 1 and 2 over $\mathbb{K}$ by Lemma 2.2 . Hence $S^{\prime}$ also does not contain 
points of degree 1 and 2 over $\mathbb{K}$ by Theorem 2.1 . Therefore, the required assertion follows from Corollary 6.6.

Corollary 7.3. Let $S$ be a smooth cubic surface over a field $\mathbb{K}$ of characteristic zero with $S(\mathbb{K})=\varnothing$ and $\operatorname{rk} \operatorname{Pic}(S)=1$. Then every finite subgroup of $\operatorname{Bir}(S)$ is abelian.

Proof. By Theorem 2.10, one has $\operatorname{Bir}(S)=\operatorname{Aut}(S)$. Thus the assertion follows from Lemma 5.5 .

Remark 7.4. For an alternative proof of Corollary 7.3 that does not use the last assertion of Theorem 2.10, one can argue as follows. Regularizing the action of $G$ and running a $G$ Minimal Model Program, we obtain a surface $S^{\prime}$ with an action of $G$ and a $G$-equivariant birational map $S \rightarrow S^{\prime}$, such that $S^{\prime}$ is either a del Pezzo surface, or a conic bundle. By Corollary 2.11, we have $S^{\prime} \cong S$. Thus the assertion follows from Lemma 5.5.

Finally, we complete the proof of Theorem 1.4. Assertion (i) is given by Lemma 5.5. Furthermore, we know from Lemma 2.9(i) that $\operatorname{rk} \operatorname{Pic}(S) \leqslant 3$. Thus, assertion (ii) is implied by Corollaries 7.1, 7.2, and 7.3 in the cases when $\operatorname{rk} \operatorname{Pic}(S)$ equals 3, 2, and 1, respectively. Assertion (iii) is given by Corollary 7.1 .

\section{REFERENCES}

[BK86] E. Brieskorn, H. Knörrer. Plane algebraic curves. Birkhäuser Verlag, Basel, 1986.

[Ca72] R. Carter. Conjugacy classes in the Weyl group. Compositio Math. 25 (1972), 1-59.

[Ch05] I. Cheltsov. Birationally rigid Fano varieties. Russian Math. Surveys 60 (2005), no. 5, 875965.

[Co77] D. Coray. Points algébriques sur les surfaces de del Pezzo. C. R. Acad. Sci. Paris Sér. A-B 284 (1977), no. 24, A1531-A1534.

[Do12] I. Dolgachev. Classical algebraic geometry. A modern view. Cambridge University Press, Cambridge, 2012.

[DI09] I. Dolgachev, V. Iskovskikh. Finite subgroups of the plane Cremona group. Algebra, arithmetic, and geometry: in honor of Yu. I. Manin. Vol. I, 443-548, Progr. Math. 269, Birkhäuser Boston, Boston, MA, 2009.

[EKM08] R. Elman, N. Karpenko, A. Merkurjev. The algebraic and geometric theory of quadratic forms. AMS Colloquium Publications, 56. American Mathematical Society, Providence, RI, 2008.

[GSh18] S. Gorchinskiy, C. Shramov. Unramified Brauer group and its applications. Translations of Mathematical Monographs, 246. American Mathematical Society, Providence, RI, 2018.

[I96] V. Iskovskikh. Factorization of birational mappings of rational surfaces from the point of view of Mori theory. Russian Math. Surveys 51 (1996), no. 4, 585-652.

[Ko16] J. Kollár. Severi-Brauer varieties; a geometric treatment. arXiv:1606.04368 (2016)

[Ma86] Yu. Manin. Cubic forms. Algebra, geometry, arithmetic. Second edition. North-Holland Mathematical Library, 4. North-Holland Publishing Co., Amsterdam, 1986.

[Po14] V. Popov. Jordan groups and automorphism groups of algebraic varieties. Automorphisms in birational and affine geometry, 185-213, Springer Proc. Math. Stat. 79, Springer, Cham, 2014.

[Sh20a] C. Shramov. Birational automorphisms of Severi-Brauer surfaces. Sb. Math., 211 (2020), no. $3,466-480$.

[Sh20b] C. Shramov. Non-abelian groups acting on Severi-Brauer surfaces. arXiv:2006.01214 (2020)

[ShV18] C. Shramov, V. Vologodsky. Automorphisms of pointless surfaces. arXiv:1807.06477 (2018)

[T16] A. Trepalin. Quotients of cubic surfaces. Eur. J. Math. 2 (2016), no. 1, 333-359.

[VA13] A. Várilly-Alvarado. Arithmetic of del Pezzo surfaces. Birational geometry, rational curves, and arithmetic, 293-319, Simons Symp., Springer, Cham, 2013. 
[Ya17] E. Yasinsky. The Jordan constant for Cremona group of rank 2. Bull. Korean Math. Soc. 54 (2017), no. 5, 1859-1871.

Steklov Mathematical Institute of Russian Academy of Sciences, 8 Gubkina st., Moscow, 119991, Russia

E-mail address: costya.shramov@gmail.com 